\title{
Multidimensional Shaping of Spatiotemporal Waves in Multimode Nonlinear Fibers
}

\author{
Stefan Wabnitz ${ }^{1}$, Senior Member, IEEE, Alioune Niang ${ }^{2}$, Daniele Modotto ${ }^{2}$, Alain \\ Barthélémy $^{3}$, Alessandro Tonello ${ }^{3}$, Vincent Couderc ${ }^{3}$, Vincent Kermene ${ }^{3}$, Agnés \\ Desfarges-Berthelemot ${ }^{3}$, Marc Fabert ${ }^{3}$, Etienne Deliancourt ${ }^{3}$, Katarzyna Krupa ${ }^{4}$, Guy Millot ${ }^{4}$ \\ ${ }^{1}$ DIET, Sapienza University of Rome, Via Eudossiana 18, 00184 Rome, Italy \\ ${ }^{2}$ Dipartimento di Ingegneria dell'Informazione, Università di Brescia, via Branze 38, 25123, \\ Brescia, Italy \\ ${ }^{3}$ Université de Limoges, XLIM, UMR CNRS 7252, 123 Avenue A. Thomas, 87060 Limoges, France \\ ${ }^{4}$ Université Bourgogne Franche-Comté, ICB UMR CNRS 6303, 9 Avenue A. Savary, 21078 Dijon, \\ France \\ Tel: (39) 06 44585669, e-mail: stefan.wabnitz@uniromal.it
}

\begin{abstract}
Recent experiments have shown that nonlinear wave propagation in multimode optical fibers leads to complex spatio-temporal phenomena. In this talk, we introduce new approaches for the control and optimization of nonlinear beam reshaping in the spatial, temporal and spectral dimensions. The first approach applies to spatial beam self-cleaning the technique of transverse wavefront shaping, which permits to launch an optimized input mode combination, that results in the stable generation of a whole nonlinear mode alphabet at the fiber output. The second approach introduces a longitudinal tapering of the core diameter of multimode active and passive fibers, which permits to generate ultra-wideband and high brightness supercontinuum light, featuring high spatial beam quality.
\end{abstract}

Keywords: nonlinear fiber optics, Kerr effect, transverse effects, supercontinuum generation, fiber amplifiers.

\section{INTRODUCTION}

It is well known that, because of random linear mode coupling, multimode optical fibers (MMFs) cannot maintain the quality of diffraction-limited light beams. Instead, a MMF spreads the energy of the beam among its many transverse modes, which unavoidably leads to a severe loss of beam quality, and the emergence of a speckled output spatial beam profile [1].

Only recently, experiments have unexpectedly demonstrated that, because of their third-order or Kerr nonlinearity, GRIN MMFs may permit the ultrafast transverse beam manipulation of high-power laser pulses. Indeed, the Kerr effect is the driving mechanism that overcomes speckle distortions at the MMF output, leading to a spatially clean, bell-shaped output beam with a diameter close to that of the fundamental mode of the MMF, which is robust against fibre bending and squeezing. The first manifestation of spatial beam self-cleaning effect involved quasi continuous-wave (CW) pulses, with temporal durations between 30 and $900 \mathrm{ps}$, and peak powers of the order of $10 \mathrm{~kW}$ [2], [3]. A similar self-cleaning process was demonstrated with femtosecond pulses in the normal dispersion regime of a GRIN MMF, albeit at more than an order of magnitude higher peak powers [4]. Kerr beam self-compression is accompanied by significant nonlinear polarization rotation of the beam [5]. Both effects can be useful for saturable absorber action in mode-locked fiber lasers, when combined with a spatial filter or an intra-cavity polarizer.

In this work, we present recent results that permit to significantly extend the flexibility of the light-activated beam control in MMFs. Namely, the self-cleaning of higher-order modes (HOMs), and the longitudinal switching of the self-cleaned mode. These two functionalities are enabled by adaptive transverse wavefront shaping on the one hand, and by engineering an active MMF taper with non-monotonic variation of the pulse energy profile along the fiber axis (dissipative landscape), on the other hand.

\section{MANY-MODE SELF-CLEANING}

For many applications, including multi-photon imaging in nonlinear microscopy and endoscopy, it is necessary to focus and 3D scan across a sample a pulsed beam at the output of a MMF. As a first step to achieve flexible nonlinear focusing from a MMF by simply adjusting the input injection conditions into the fiber, Deliancourt et al. demonstrated Kerr beam self-cleaning of HOMs in a GRIN MMF. By appropriately tilting the input beam, output beam profiles different from a bell-shape could be generated, for example including the $L P_{11}$ and $L P_{21}$ modes [6]. This proof-of-principle experiment has however the drawback of requiring a manual control of the input launching conditions, which is difficult to precisely replicate.

To overcome this difficulty, Deliancourt et al. [7] developed a scheme of optimized adaptive wavefront shaping of the input coherent beam (see Fig. 1). A wavefront shaping approach has recently been introduced by Tzang et al. to control, by a spatial light modulator at the fibre input coupled with real-time spectral feedback and genetic 


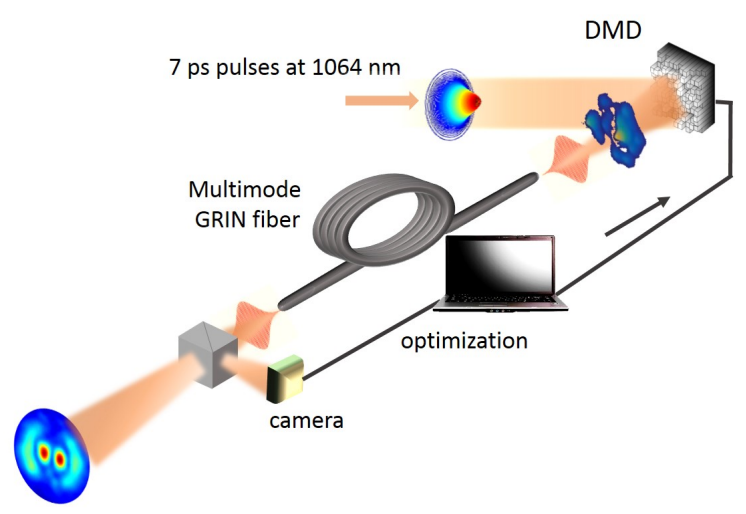

Figure 1: Scheme of wavefront shaping setup.

algorithm optimization, the efficiency of stimulated Raman scattering cascade and inter-modal four-wave mixing in a MMF [8]. In the experiments by Deliancourt et al., the adaptive beam profiling method led to generating the Kerr beam self-cleaning of many low-order modes in a GRIN MMF, by exploiting nonlinear coupling among all guided modes. They used 7 ps pulses at $1064 \mathrm{~nm}$, injected in a five meters long MMF operating in the normal dispersion regime. Optimized self-cleaning of five different LP modes was obtained, with a power threshold growing larger with the mode order [7]. Figure 2 shows examples of different output mode profiles, obtained in correspondence of different phase maps on the pixels of a deformable mirror device (DMD) at the fiber input. The DMD was controlled by a computer and a feedback loop involving an error signal generated by computing the difference between the actual fiber output pattern and the target (or objective) one.
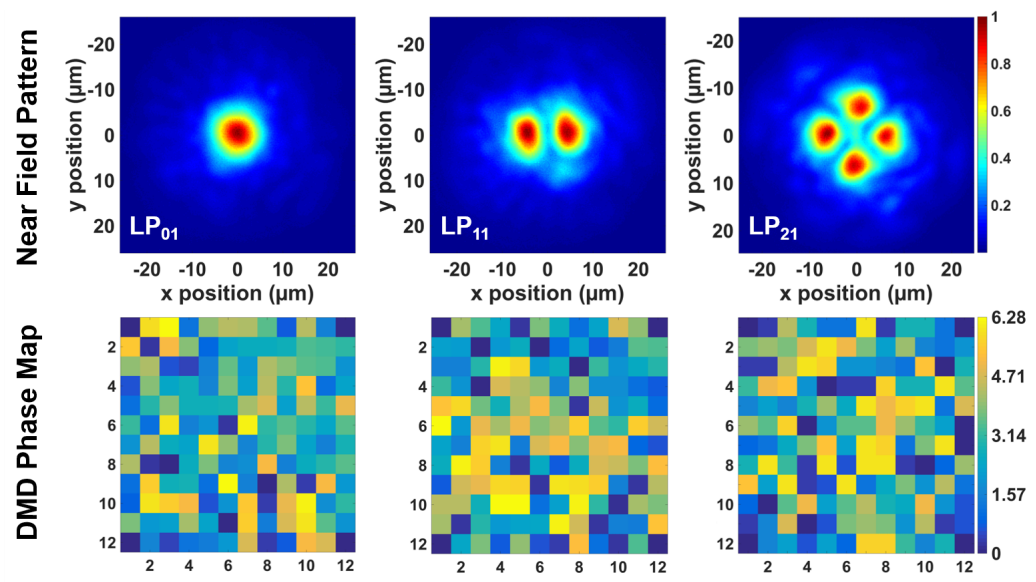

Figure 2: Different self-cleaned modes and their corresponding DMD pixel patterns.

\section{DISSIPATIVE LANDSCAPE}

Since multimode active fibers are important for the up-scaling of the output power from fiber optic amplifiers and lasers, we investigated in a series of works the possibility of Kerr self-cleaning in different types of active MMFs. Previously, Guenard et al. obtained relatively low input power threshold self-cleaning with a doubleclad $\mathrm{Yb}$ doped highly MMF with nearly step-index refractive index profile and uniform over the core doping profile [9]. Guenard et al. also studied self-cleaning in a composite cavity laser configuration, where a passively Q-switched Nd:YAG microchip laser was combined with an external cavity including the same multimode Ybdoped MMF [10]. For appropriate coupling levels with the extended cavity, beam self-cleaning could be observed in the active MMF, leading to a quasi-single mode transverse output, and up to two-fold temporal compression of the laser pulses. Spatial self-cleaning was accompanied by the observation of far-detuned nonlinear intra-cavity frequency conversion.

In order to further expand the possibilities of controlling the spatial and spectral shaping of multimode beams, we developed an Yb-doped MMF taper, with parabolic index and doping profiles in the core, whose diameter is exponentially decreasing along the $10 \mathrm{~m}$ long taper from $120 \mu \mathrm{m}$ to $40 \mu \mathrm{m}$. Tapering leads to an acceleration of the self-imaging oscillations in the MMF: Niang et al. directly measured the self-imaging period along the 


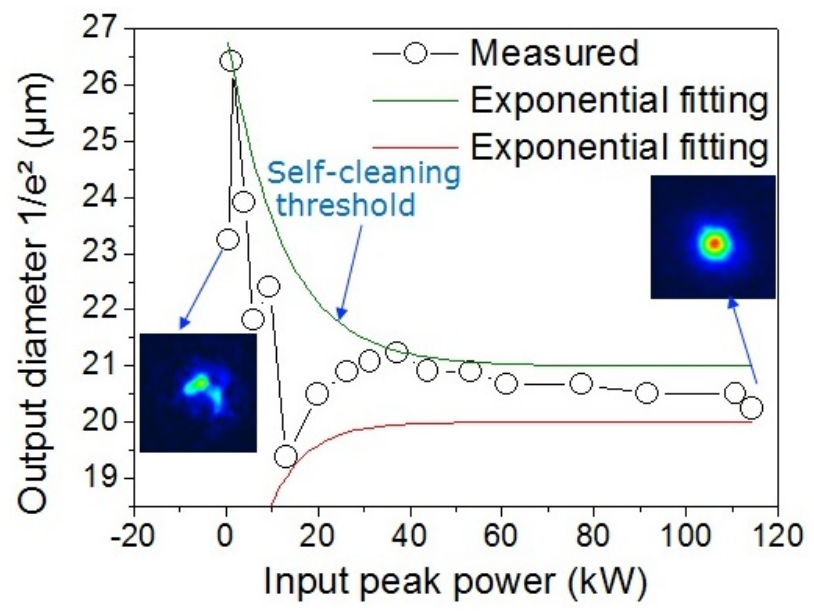

Figure 3: Experimental diameter of near-field output beam vs. input peak power.

MMF taper, and obtained that it decreases from $1.2 \mathrm{~mm}$ at the large core diameter end, down to $360 \mu \mathrm{m}$ at the small diameter side [11].

First we studied self-cleaning in the lossy Yb-doped taper, that is in the absence of a diode pump. Figure 3 shows the dependence of the output beam diameter vs. input peak power. As can be seen, the output beam diameter fluctuates wildly at low powers, while for peak powers above $\simeq 20 \mathrm{~kW}$ it stabilizes to about $20.5 \mu \mathrm{m}$.

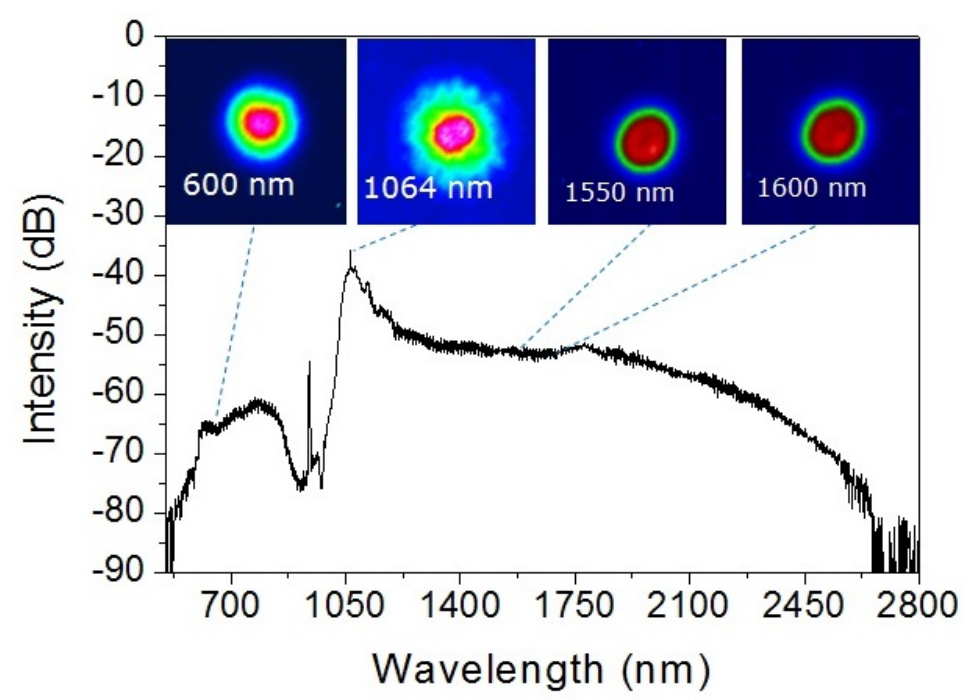

Figure 4: Output supercontinuum generation from pumped active taper, and corresponding bell-shaped beam profiles.

In the presence of gain induced by a laser diode pump, the accelerating self-imaging leads to a continuous up-shift of the anti-Stokes geometric parametric instability (GPI) sidebands along the taper [11], which permits to broaden into the visible region the supercontinuum (SC) generated at the taper output (see Fig. 4), extending between 520 and $2600 \mathrm{~nm}$. At the same time, Fig. 4 shows that high beam quality is maintained across the SC spectral width.

As shown by Fig. 5, when pumping the Yb-doped MMF from the large diameter side, gain is only present over the first couple of meters. After that, the signal experiences a loss. By taper cut-back, we have revealed that this dissipative landscape leads to the longitudinal switching of different self-cleaned modes. After the first $2 \mathrm{~m}$ of taper, where SC generation occurs, a self-cleaned $L P_{11}$-like mode is observed. Subsequently, in the regime where linear attenuation prevails, a bell-shaped beam resembling the fundamental $L P_{01}$ mode of the taper is generated.

\section{CONCLUSIONS}

Multimode nonlinear fiber optics is a promising platform for the up-scaling of the energy of coherent fiber laser sources, and the ultrafast control of the spatiotemporal content of laser pulses. We reported two novel strategies, 


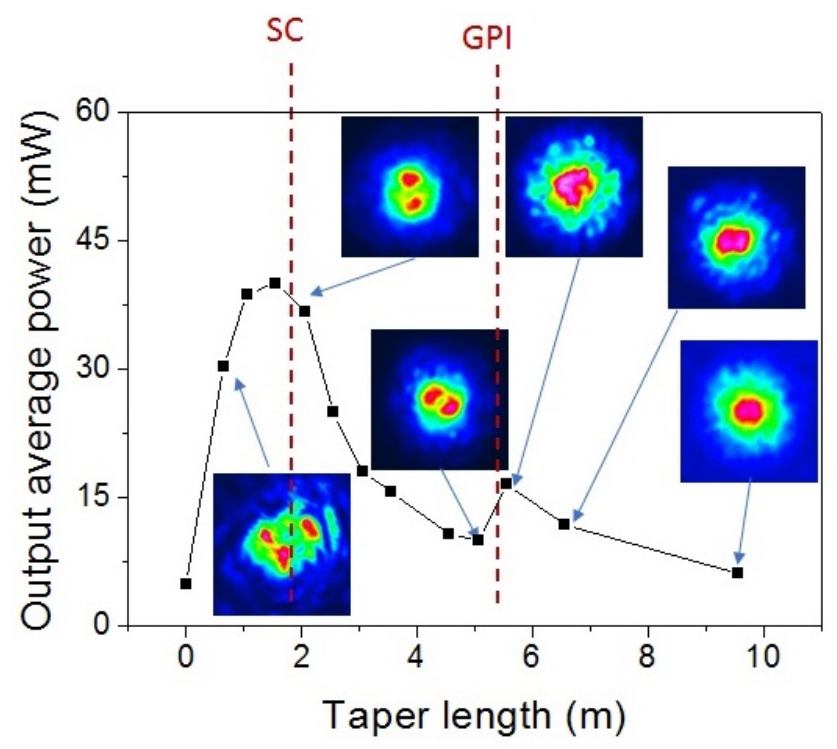

Figure 5: Measured output average power vs. taper length, and corresponding beam profiles.

adaptive wavefront control and dissipative tapering, to enable the robust and flexible control of the transverse and spectral properties of multimode light waves.

\section{ACKNOWLEDGMENTS}

This work was supported by the European Research Council (ERC) under the European Union's Horizon 2020 research and innovation programme (grant No. 740355).

\section{REFERENCES}

[1] D. Gloge, "Optical power flow in multimode fibers," Bell System Technical Journal, vol. 51, no. 8, pp. 1767-1783, 1972.

[2] K. Krupa, A. Tonello, B. M. Shalaby, M. Fabert, A. Barthélémy, G. Millot, S. Wabnitz, and V. Couderc, "Spatial beam self-cleaning in multimode fibres," Nat. Photonics, vol. 11, pp. 234-241, 2017.

[3] L. G. Wright, Z. Liu, D. A. Nolan, M.-J. Li, D. N. Christodoulides, and F. W. Wise, "Self-organized instability in graded-index multimode fibres," Nat. Photonics, vol. 10, pp. 771-776, November 2016.

[4] Z. Liu, L. G. Wright, D. N. Christodoulides, and F. W. Wise, "Kerr self-cleaning of femtosecond-pulsed beams in graded-index multimode fiber," Opt. Lett., vol. 41, no. 16, pp. 3675-3678, Aug 2016.

[5] K. Krupa, G. G. Castañeda, A. Tonello, A. Niang, D. S. Kharenko, M. Fabert, V. Couderc, G. Millot, U. Minoni, D. Modotto, and S. Wabnitz, "Nonlinear polarization dynamics of Kerr beam self-cleaning in a graded-index multimode optical fiber," Opt. Lett., vol. 44, no. 1, pp. 171-174, Jan 2019.

[6] E. Deliancourt, M. Fabert, A. Tonello, K. Krupa, A. Desfarges-Berthelemot, V. Kermene, G. Millot, A. Barthélémy, S. Wabnitz, and V. Couderc, "Kerr beam self-cleaning on the LP11 mode in graded-index multimode fibers," OSA Continuum, vol. 2, no. 4, pp. 1089-1096, Apr 2019.

[7] E. Deliancourt, M. Fabert, A. Tonello, K. Krupa, A. Desfarges-Berthelemot, V. Kermene, G. Millot, A. Barthélémy, S. Wabnitz, and V. Couderc, "Wavefront shaping for optimized many-mode Kerr beam self-cleaning in graded-index multimode fiber," arXiv e-prints, p. arXiv:1902.04453, Feb 2019.

[8] O. Tzang, A. M. Caravaca-Aguirre, K. Wagner, and R. Piestun, "Adaptive wavefront shaping for controlling nonlinear multimode interactions in optical fibres," Nat. Photonics, vol. 12, pp. 368-374, May 2018.

[9] R. Guenard, K. Krupa, R. Dupiol, M. Fabert, A. Bendahmane, V. Kermene, A. Desfarges-Berthelemot, J. L. Auguste, A. Tonello, A. Barthélémy, G. Millot, S. Wabnitz, and V. Couderc, "Kerr self-cleaning of pulsed beam in an ytterbium doped multimode fiber," Opt. Express, vol. 25, no. 5, pp. 4783-4792, Mar 2017.

[10] — - "Nonlinear beam self-cleaning in a coupled cavity composite laser based on multimode fiber," Opt. Express, vol. 25, no. 19, pp. 22 219-22 227, Sep 2017.

[11] A. Niang, T. Mansuryan, K. Krupa, A. Tonello, M. Fabert, P. Leproux, D. Modotto, G. Millot, V. Couderc, and S. Wabnitz, "Spatial Beam Self-Cleaning and Supercontinuum Generation with Yb-doped Multimode Graded-Index Fiber Taper Based on Accelerating Self-Imaging and Dissipative Landscape," arXiv e-prints, p. arXiv:1904.03224, Apr 2019. 